\title{
DIFFUSION OF ANTIBIOTICS FROM ACRYLIC BONE-CEMENT IN VITRO
}

\author{
Joyce Hill, L. Klenerman, Sheila Trustey and R. Blowers, Harrow, England \\ From the Clinical Research Centre and Northwick Park Hospital, Harrow
}

\begin{abstract}
The diffusion of Fucidin, gentamicin, and clindamycin from acrylic cement was tested in an in vitro system. The activity of Fucidin was very short-lived and only against gram-positive organisms; gentamicin inhibited gram-positive and gram-negative organisms for twenty-two and eleven days respectively; clindamycin had significant action only against gram-positive organisms and retained some activity for fifty-six days.

We suggest that the destruction of organisms in the tissues is more likely to be achieved by topical and intravenous administration of antibiotics during the operation than by incorporation of antibiotic in the cement.
\end{abstract}

Deep wound infection is a serious complication of hip joint replacement. Whether early or late in appearance, such infection is presumed to be due to wound contamination during the operation. The prevention of infection must depend mainly on aseptic and atraumatic techniques and the avoidance of airborne infection during operations; despite such measures, contamination may still occur.

The value of antibiotics in preventing wound infection is much argued, but many, if not most, surgeons use prophylactic antibiotics for hip replacement operations. The antibiotics are usually given systemically from just before the operation and for a few days after, and by local application in the wound usually towards the end of the operation. More recently Buchholz and Engelbrecht (1970) suggested the incorporation of antibiotic in the acrylic cement employed to fix the prosthesis to the bone; the claimed advantage of this method is the release of antibiotic into the surrounding tissues over a long period. They incorporated gentamicin in "Palacos" cement and reported in vitro experiments in which the antibiotic was released continuously, in decreasing amount, for about two months. Medcraft and Gardner (1974) incorporated 250 milligrams of Fucidin in $\mathbf{4 0}$ grams of "Simplex P" cement (6.25 milligrams per gram) and found that discs of this mixture, placed on bacteriological culture medium sown with staphylococci, become surrounded by a zone of inhibition; the discs retained this property during dry storage for at least twelve months.

Levin (1975) immersed "Simplex B" cement containing an antibiotic (37.5 milligrams per gram) in saline at 37 degrees Celsius to simulate the leaching effect of body fluids. Residual antibacterial properties were assessed by measuring the zone of bacterial inhibition around them. Clindamycin was able to inhibit grampositive, but not gram-negative, organisms for more than sixty days, and cephalothin for about twenty days, but the activity of gentamicin, erythromycin, tetracycline, and methicillin could not be detected after only a few days; Fucidin was not tested.

We have chosen to test gentamicin because of its wide antibacterial spectrum and the prolonged activity reported by Buchholz and Engelbrecht (1970), and because, unlike many antibiotics, it is stable at the temperature (up to 120 degrees Celsius (Jefferiss, Lee and Ling 1975)) reached during the curing of polymethylmethacrylate. Fucidin was selected because of the favourable report by Medcraft and Gardner (1974), and clindamycin on account of its superior performance in the experiments of Levin (1975).

Our technique was similar to that of Levin (1975). We think that it more nearly reproduces conditions in the tissue than does the dry-storage method of Medcraft and Gardner (1974).

\section{MATERIALS AND METHODS}

Cement discs-Five batches of "Simplex P" cement were made up, each in standard 40-gram amounts, to which we added antibiotics as follows: Fucidin 200 milligrams ( 5 milligrams per gram of cement) and 8 grams (200 milligrams per gram); gentamicin 500 milligrams (12.5 milligrams per gram) and 8 grams (200 milligrams per gram); and clindamycin 1.5 grams (37.5 milligrams per gram). From each batch, discs about 10 millimetres diameter, 4 millimetres thick, and weighing about 1 gram were made. The discs were stored dry at 4 degrees Celsius until required.

Joyce Hill, F.I.M.L.T., Division of Hospital Infection, Clinical Research Centre, Harrow HA1 3UJ, England.

L. Klenerman, Ch.M., F.R.C.S., Northwick Park Hospital, Harrow HA1 3UJ, England.

Sheila Trustey, B.Sc., Division of Hospital Infection, Clinical Research Centre, Harrow HA1 3UJ, England.

R. Blowers, M.D., F.R.C.P., F.R.C.Path., Division of Hospital Infection, Clinical Research Centre, Harrow HA1 3UJ, England. 
Organisms - Three organisms were used : staphylococcus aureus (Oxford strain) NCTC no. 6571, staphylococcus albus, and E. coli (local hospital strains). By conventional disc tests the staphylococci were fully sensitive to Fucidin, gentamicin, and clindamycin; the $\mathrm{E}$. coli was sensitive to gentamicin, but resistant to Fucidin and clindamycin.

TABLE I

Antibacterial Effect of "Simplex" Disc Containing Fucidin 5 Milligrams per Gram of Cement

\begin{tabular}{|l|c|c|c|}
\hline \multirow{2}{*}{ Organism } & \multicolumn{2}{|c|}{$\begin{array}{c}\text { Growth in peptone } \\
\text { water after days }\end{array}$} & $\begin{array}{c}\text { Width of inhibition } \\
\text { zone on agar } \\
\text { after eight days } \\
\text { (millimetres) }\end{array}$ \\
\cline { 2 - 4 } & $\mathbf{3}$ & $\mathbf{7}$ & $\mathbf{0}$ \\
\hline Staphylococcus aureus & - & + & 0 \\
Staphylococcus albus . & - & + & $\mathbf{0}$ \\
E. coli . & + & $\cdots$ & 0 \\
\hline
\end{tabular}

$-=$ no growth $;+=$ growth; $\ldots=$ not tested.

Tests of antibacterial activity-Discs of antibiotic-containing cement were put into screw-capped bottles containing 10 millilitres of peptone water. After two hours, the peptone water in each bottle was inoculated with one drop of an overnight broth culture of the relevant test organism. The bottles were incubated at 37 degrees Celsius and were examined for turbidity twice weekly. When no bacterial growth occurred the disc was removed, washed three times in peptone water and put in a fresh bottle of peptone water which was inoculated and incubated as before. This process was repeated twice weekly until bacterial growth occurred. longer seen. We used this method because preliminary tests had shown residual activity in the disc after it no longer prevented growth in the liquid medium.

\section{RESULTS}

Table I shows the results from discs containing 5 milligrams of Fucidin per gram of cement. In the liquid medium staphylococci were inhibited at three days but had grown at seven days; transfer of the disc to the more sensitive agar-diffusion test revealed no further activity. E. coli grew freely from the beginning, as would be expected. Tests on discs containing 200 milligrams of Fucidin per gram of cement showed no significant difference.

Table II shows the results for gentamicin impregnated discs containing 12.5 milligrams of gentamicin per gram of cement. Inhibition of staphylococcus aureus had ceased at seventeen days, but continued feebly on solid medium for another five days-twenty-two days in all. Staphylococcus albus and E. coli were inhibited in liquid medium for seven days and on solid medium until the eleventh day. Increasing the concentration of gentamicin to 200 milligrams per gram did not extend the period of activity.

Table III shows the results for clindamycin impregnated discs containing 37.5 milligrams per gram. Only staphylococcus aureus and E. coli were included in these

TABLE II

Antibacterial Effect of "Simplex" Disc Containing Gentamicin 12.5 Milligrams Per Gram of Cement

\begin{tabular}{|c|c|c|c|c|c|c|c|c|c|}
\hline \multirow[b]{2}{*}{ Organism } & \multicolumn{5}{|c|}{ Growth in peptone water after days } & \multicolumn{4}{|c|}{$\begin{array}{l}\text { Width of inhibition zone on agar } \\
\text { after additional days } \\
\text { (millimetres) }\end{array}$} \\
\hline & 3 & 7 & 10 & 14 & 17 & 1 & 4 & 5 & 6 \\
\hline Staphylococcus aureus & - & - & - & - & + & 2 & 2 & 2 & 0 \\
\hline Staphylococcus albus. & - & - & + & $\cdots$ & $\cdots$ & 13 & $\mathbf{0}$ & $\cdots$ & $\cdots$ \\
\hline E. coli & - & - & + & $\ldots$ & $\ldots$ & 3 & 0 & $\ldots$ & $\ldots$ \\
\hline
\end{tabular}

$-=$ no growth; $+=$ growth; $\ldots=$ not tested.

TABLE III

Antibacterial Effect of "Simplex" Disc Containing Clindamycin 37.5 Milligrams per Gram of Cement

\begin{tabular}{|c|c|c|c|c|c|c|c|c|c|c|c|c|c|c|}
\hline \multirow[b]{2}{*}{ Organism } & \multicolumn{10}{|c|}{ Growth in peptone water after days } & \multicolumn{4}{|c|}{$\begin{array}{l}\text { Width of inhibition zone } \\
\text { after additional days } \\
\text { (millimetres) }\end{array}$} \\
\hline & 3 & 7 & 10 & 14 & 17 & 21 & 24 & 28 & 31 & 36 & 1 & 4 & 5 & 20 \\
\hline Staphylococcus aureus & - & - & - & - & - & - & - & - & - & + & 8 & 11 & 9 & 9 \\
\hline E. coli & - & + & ... & $\ldots$ & $\ldots$ & $\ldots$ & $\ldots$ & $\ldots$ & $\ldots$ & $\ldots$ & 0 & $\ldots$ & $\ldots$ & $\ldots$ \\
\hline
\end{tabular}

$-=$ no growth; $+=$ growth; $\ldots=$ not tested.

From bottles in which bacterial growth had occurred the cement discs were removed, washed three times, and retested for activity against the same organism by a diffusion method on a solid medium. Each disc was placed on the surface of Sensitest Agar (Oxoid Ltd.) that had been flooded with an overnight culture of the test organism and dried. The plates were incubated for eighteen hours at $\mathbf{3 7}$ degrees Celsius and the inhibition zones were measured from the edge of the disc to the edge of the bacterial growth. This process was repeated daily, except at week-ends, until a zone of inhibition was no tests. Staphylococcus aureus was inhibited in liquid medium for fourteen days and on solid medium at least until the fifty-sixth day when the tests were discontinued. E. coli was inhibited for only three days.

\section{DISCUSSION}

For the purpose of assaying the release of antibiotic it is obviously impossible to reproduce, in vitro, the conditions 
to which acrylic cement is exposed in the human body. However, it seemed to us that ihe leaching effect of tissue fluids and the blood stream would be better simulated by immersion of the cement in a liquid than by dry storage. We therefore used, as our main indication of activity, the inhibitory action of the cement plus antibiotic discs in a small volume of liquid culture medium, which was changed and reinoculated with test organisms twice a week; a continuous flow of liquid, to simulate the blood stream, might have leached away the antibiotic more quickly. The test on the solid medium was more sensitive and indicated a possible extension of antibacterial activity.

If an antibacterial drug is to prevent sepsis after a hip operation, it might be required to act in two ways: a) by achieving a broad-spectrum bactericidal concentration in the exposed tissues during the operation, so that contaminating organisms are killed instantly; and $b$ ) by maintaining a bactericidal concentration to deal with blood-borne micro-organisms that might later colonise devitalised tissues around che implant.

Antibiotic impregnated cement cannot fulfil the first requirement because it is not implanted until near the end of the operation; diffusion of antibiotics from cement is unlikely to create a significant bactericidal zone until several hours have elapsed. This is more likely to be achieved by the direct application of antibiotic powder or solution to the wound. Intravenous injection of antibiotic has been shown to give almost immediate bactericidal concentrations of oxacillin, cephaloridine, clindamycin and methicillin in blood, bone, synovial capsule and fluid, and in haematomas (Bowers, Wilson and Greene 1973; Kolczun, Nelson, McHenry, Gavan and Pinovich 1974; Hughes, Benson, Dash and Field 1975; Schurman, Johnson, Finerman and Amstutz 1975).

Of the antibiotics that we and others have tested in vitro, none seems likely to fulfil the second requirement without risk to the patient. The activity of Fucidin was short-lived. Either it was rapidly leached out or it escaped only from the superficial parts of the cement mass. Moreover, its limited antibacterial spectrum makes it a poor choice for protection against infection. Gentamicin has a wider bactericidal spectrum and, in our experiment, had a more prolonged action. However, human hypersensitivity to gentamicin has been recorded (Pirilä, Förström and Rouhunkoski 1967); if this occurred to a patient with gentamicin in the bone cement, the prosthesis would have to be removed. Clindamycin discs showed prolonged activity against staphylococci but, as with Fucidin, the narrow antibacterial spectrum is a disadvantage.

It seems to us that the practice of incorporating antibiotic in bone cement does not rest on sound principles. The main requirement, instant killing during the operation, is achieved more certainly by topical and intravenous administration. The need for prolonged activity depends on the unproven role of blood-borne infection in the causation of peri-articular infection. We do not join the discussion on whether or not antibiotics should be used prophylactically for hip replacement operations, but suggest that if they are employed, intravenous and topical administration during the operation are more likely to succeed than incorporation of an antibiotic in the bone cement.

\section{REFERENCES}

Bowers, W. H., Wilson, F. C., and Greene, W. B. (1973) Antibiotic prophylaxis in experimental bone infections. Journal of Bone and Joint Surgery, 55-A, 795-807.

Buchholz, H. W., and Engelbrecht, H. (1970) Uber die Depotwirkung einiger Antibiotica bei Vermischung mit dem Kunstharz Palacos. Chirurg, 41, 511-515.

Hughes, S. P. F., Benson, M. K. D., Dash, C. H., and Field, C. A. (1975) Cephaloridine penetration into bone and synovial capsule of patients undergoing hip joint replacement. Journal of Antimicrobial Chemotherapy, 1 (3 Suppl.), 41-46.

Jefferiss, C. D., Lee, A. J. C., and Ling, R. S. M. (1975) Thermal aspects of self-curing polymethylmethacrylate. Journal of Bone and Joint Surgery, 57-B, 511-518.

Kolczun, M. C., Nelson, C. L., McHenry, M. C., Gavan, T. L., and Pinovich, P. (1974) Antibiotic concentrations in human bone. Journal of Bone and Joint Surgery, 56-A, 305-310.

Levin, P. D. (1975) The effectiveness of various antibiotics in methylmethacrylate. Journal of Bone and Joint Surgery, 57-B, $234-237$.

Medcraft, J. W., and Gardner, A. D. H. (1974) The use of an antibiotic bone cement combination as a different approach to the elimination of infection in total hip replacement. Medical Laboratory Technology, 31, 347-353.

Pirilä, V., Förström, L., and Rouhunkoski, S. (1967) Twelve years of sensitization to neomycin in Finland. Acta Dermato-venereologica, $47,419-425$.

Schurman, D. J., Johnson, B. L., Jun., Finerman, G., and Amstutz, H. C. (1975) Antibiotic bone penetration. Clinical Orthopaedics and Related Research, 111, 142-146. 